\title{
Matériaux désordonnés, verres et leur fonctionnalisation
}

\author{
Lionel Montagne ${ }^{1}$, Wilfried Blanc ${ }^{2}$ et Daniel R. Neuville ${ }^{3}$
}

\author{
Reçu le 10 juillet 2015, accepté le 10 juillet 2015
}

\begin{abstract}
Résumé - Ce numéro thématique présente des articles qui illustrent la diversité des activités de recherche et développement menées sur les verres en France, et reflètent bien l'esprit avec lequel le GDR « Verres » et l'Union pour la Science et la Technologie Verrières (USTV) agissent pour fédérer les actions scientifiques sur les verres pour le GDR et pour et assurer un lien entre les communautés académiques et industrielles.

Abstract - Disordered materials, glasses and their functionalization. This Topical Issue presents articles which illustrate the diversity of the activities of research and development led on glasses in France, and reflects the way in which the GDR "Glass" and the Union for Glass Science and Technology (USTV) act to federate the scientific actions on glasses for the GDR and to insure the link between the academic and industrial communities.
\end{abstract}

Le GDR Verres (GDR CNRS 3338) et l'Union pour la Science et la Technologie Verrières (USTV) s'associent pour publier ce numéro thématique de Matériaux et Techniques consacré aux matériaux vitreux. Les articles sont, pour la plupart, écrits sur la base des présentations dans les conférences présentées au congrès Matériaux-2014 de Montpellier, où le GDR et l'USTV ont co-organisé un symposium intitulé « Matériaux désordonnés, verres et leur fonctionnalisation $»$.

Le symposium a rassemblé une centaine de participants dont un quart de doctorants dont l'inscription a été financée par le GDR. La présidence scientifique en a été assurée par Étienne Vernaz du CEA, Lionel Montagne, Directeur du GDR-Verres, et Daniel Neuville, Président de l'USTV.

Une première session était consacrée aux verres de chalcogénures et aux verres d'optique. Présidée par Annie Pradel de l'université de Montpellier, elle a été introduite par une conférence invitée d'Andrea Piarristeguy, faisant le point sur les propriétés spécifiques de ce type de verre. Huit exposés oraux ont porté sur le développement d'optiques adaptées à la vision nocturne, de capteurs infrarouge, de fibres destinées à la détection du $\mathrm{CO}_{2}$, de matériaux microstructurés aux propriétés optiques optimisées, ou encore sur des matériaux hybrides formés d'un liquide ionique confiné dans un chalcogénure nanoporeux.

La seconde session, intitulée « Verres et amorphes dans tous les états », était présidée par Etienne Vernaz du CEA. Elle a été introduite par une conférence invitée d'Anne-Lise Faivre qui portait sur la définition du verre et de la transition vitreuse. Même si ce point est débattu depuis plus d'un siècle, on constate que « l'état vitreux » garde encore sa part de mystère, ouvrant un champ toujours renouvelé à la recherche, aussi bien fondamentale qu'appliquée. Dix présentations orales ont permis d'en explorer quelques aspects, comme le volume libre interatomique exploré par compression avec des gaz de taille atomique différentes, ou encore l'autocicatrisation de verres composites soumis à des conditions sévères.

La dernière session, présidée par Lionel Montagne de l'université de Lille, portait sur les verres et matériaux de confinement. Elle a été introduite par un exposé général de Florence Bart, chef de projet au CEA Marcoule, et a été suivi de huit exposés portant sur la solubilité

\footnotetext{
1 UCCS, Unité de Catalyse et Chimie du Solide, UMR CNRS 8181, Université de Lille 1, École Nationale Supérieure de Chimie de Lille, Cité Scientifique, Bâtiment C7, BP 90108, 59652 Villeneuve d'Ascq Cedex, France

lionel.montagne@univ-lille1.fr

2 Université Nice Sophia Antipolis, CNRS, LPMC, UMR 7336, 06100 Nice, France

3 Géomatériaux, CNRS-Institut de Physique du Globe de Paris, Sorbonne Paris Cité, 1 rue Jussieu, 75238 Paris Cedex 05, France; Éditeur « Matériaux et Techniques »
} 
de différents éléments (lanthanides, molybdène...) dans les borosilicates ou aluminosilicates complexes (inclusion, cristallisation, séparation de phase), sur le comportement de ces verres à l'auto irradiation, sur la compréhension de la surface réactive formée dans l'eau, ou encore sur la modélisation des propriétés physico-chimiques à partir de modélisation atomistique.

Les articles sélectionnés pour ce numéro spécial illustrent la diversité des activités de recherche et développement menées sur les verres en France, et reflètent bien l'esprit avec lequel le GDR et l'USTV agissent pour fédérer les actions scientifiques sur les verres pour le GDR et pour et assurer un lien entre les communautés académiques et industrielles.

L'USTV et le GDR-Verres assurent ainsi la représentation et l'animation de la communauté scientifique verrière en partenariat avec la Fédération des Chambres Syndicales des Industries du Verre. Des actions sont régulièrement organisées, telles que des ateliers, écoles thématiques et journées sur des problématiques allant du contact alimentaire aux propriétés du verre à chaud ou aux mécanismes de nucléation et cristallisation.

Parmi les prochains évènements organisés, on peut noter les «Journées Verres » qui se tiennent à Nice du 18 et 20 novembre 2015. Elles sont précédées par un atelier thématique sur les « dégâts d'irradiation » dans les verres, les 17 et 18 novembre. Les participants ont de plus la chance de visiter les verreries de Biot et d'en admirer les productions artistiques. La photo de couverture de ce numéro de Matériaux et Techniques est une œuvre produite à Biot par Antoine Pierini, qui servira de récompense pour les prix posters décernés lors des Journées Verres.

En conclusion, si vous vous intéressez au verre, au sens le plus large du terme, que vous soyez industriel, chercheur, étudiant, artiste, ou tout simplement curieux, n'hésitez pas à nous rejoindre, toutes les informations sont sur www.ustverre.fr ou http://gdrverres. univ-lille1.fr. 